\title{
Area-filling curves
}

\author{
Maria Chiara Nasso(id And Aljoša VolČIČ (D
}

\begin{abstract}
In this paper, we study area-filling curves, i.e. continuous and injective mappings defined on $[0,1]$ whose graph has positive measure. Current literature calls them "Osgood curves", but their invention is due to H. Lebesgue. Stromberg and Tseng constructed homogeneous areafilling curves and offered an elegant example. We show that an appropriate variant of Knopp's construction attains the same homogeneity result. In Section 4, we discuss briefly the existence of an "invasive" curve, i.e. a continuous and injective mapping from the half-open interval $[0,1[$ to the unit square, whose image has measure 1 . In the last section, we discuss several aspects of the Lance-Thomas curve, connecting it with the other construction due to Stromberg and Tseng.
\end{abstract}

Mathematics Subject Classification. 26A30, 26B35.

Keywords. Osgood curves, Space-filling curves, Oxtoby-Ulam theorem.

1. Introduction. This paper is dedicated to some questions raised by Sagan's entertaining book "Space-filling curves" [18] and the deep and analytically highly non-trivial paper [21].

Peano, Hilbert $([6,15])$, and other authors constructed examples of surjective continuous mappings $f:[0,1] \rightarrow[0,1]^{2}$ which are called space-filling curves. It is well-known that such curves cannot be injective by a result of Netto [12].

Nowadays, space-filling curves have several useful and surprising applications in global optimization (see e.g. [19]).

In analogy, we shall call area-filling curves those continuous and injective mappings from $[0,1]$ to $[0,1]^{2}$ which cover a set of positive measure.

To our knowledge, there are at least ten constructions of area-filling curves. Moreover there are two existence theorems. Lebesgue and Osgood proposed two constructions which are in essence equivalent. The others are due to 
Sierpiński [20], Knopp [7], Gelbaum and Olmsted (who offered two constructions) [4], Lance and Thomas [9] (with the variant due to Sagan [17] and another one due to Ravishankar and Salas [16]), and two different constructions are due to Stromberg and Theng [21]. The last paper contains also a non-constructive existence proof suggested to the authors by Sadahiro Saeki. Another interesting non-constructive existence proof was given in [1] by Balcerzak and Kharazishvili using the Denjoy-Riesz theorem. All the results we discuss are variants of the following

Theorem 1.1. Given a regular planar set $Q$ having measure 1 , for every $\beta \in] 0,1\left[\right.$, there exists a continuous injective curve $\gamma_{\beta}:[0,1] \rightarrow Q$ such that $\lambda_{2}\left(\gamma_{\beta}([0,1])\right)=\beta$.

We indicate with $\lambda_{m}$ the $m$-dimensional Lebesgue measure. In [20] and [7], $Q$ is a triangle, in [11], it is a sector of a circular crown. In all the other constructions, $Q$ is the unit square. Sierpiński's construction has the drawback that it does not allow one to choose an arbitrary $\beta \in] 0,1[$.

Current literature consistently calls such curves "Osgood curves" but we suggest to call them "area-filling curves" since we show in Section 2 that Lebesgue invented such curves one year before Osgood. On the other hand, we cannot call them Lebesgue curves since there already exists a space-filling curve named after him.

A curve is a continuous mapping from an interval $I \subset \mathbb{R}$ into $\mathbb{R}^{m}$. In our paper, $I$ is a bounded closed interval, except in Section 4, where topological circumstances suggest us to take it half-open.

If $\varphi$ and $\psi$ are two curves defined on $[a, b]$ and $\left[a^{\prime}, b^{\prime}\right]$, respectively, with values in $\mathbb{R}^{m}$, we call them equivalent if there exists an increasing homeomorphism $s(\cdot)$ from $[a, b]$ to $\left[a^{\prime}, b^{\prime}\right]$ such that $\varphi(\cdot)=\psi(s(\cdot))$ for every $t \in[a, b]$.

Sometimes it is convenient to identify curves with their equivalence classes, while in other cases it is important to distinguish between two equivalent curves (see also [3]).

This ambivalent approach can already be seen in the papers which are at the origin of space- and area-filling curves: Peano provides the analytic expression of his curve (and shows no picture), while Hilbert only shows pictures, saying that they lead trivially to their parametric representation.

Area-filling curves are not part of the traditional program offered to students of mathematics in spite of the simplicity of some constructions and the influential mathematicians who contributed to their study. Area-filling curves tend to be forgotten and rediscovered (see [18, pp. 132-133]).

We think, on the contrary, that they should belong to the permanent display of amusing objects aimed to arouse the interest of students (like the Peano and Hilbert curve, the snowflake curve, the Cantor set and the Cantor function, the Sierpiński triangle, the Möbius strip, Klein's bottle, and the rest of the zoo). The origin itself of the area-filling curves is curious. In Section 2, we discuss their early history. In Section 3, we discuss the homogeneous constructions of area-filling curves, a concept introduced in [21]. We show that an 
appropriate (and simpler) variant of Knopp's construction also has the homogeneous property. In Section 4, we discuss a related question, the existence of a continuous and injective mapping $\varphi:\left[0,1\left[\rightarrow \mathbb{R}^{m}\right.\right.$ such that $\lambda_{m}(\varphi([0,1[))=1$. In the last section, we generalize the construction of Lance and Thomas [9] and construct a class of area-filling curves which depend (for any given $\beta$ ) on continuously many parameters. They are parametrized on symmetric Cantor sets which turn out to be homeomorphic to the essential trace of the curve.

2. Early history. H. Lebesgue, in his famous thesis, considered a measuretheoretic problem which can be reformulated in the following way: Suppose $A$ is the interior of a Jordan curve. Is A Peano-Jordan-measurable?

Putting it in other words, the question is if there are Jordan curves whose image (sometimes called trace) has positive Jordan measure.

This was unknown at that time and Lebesgue sketched in a footnote of his thesis (page 17) the general idea for the proof of their existence. It was based on the construction of the Peano curve.

Osgood [13], one year after the publication of Lebesgue's thesis, constructed independently an example of an injective curve having positive area, based on the construction of the Peano curve.

In the same year, [11] Lebesgue returned to his measure-theoretic question, elaborating a particular instance of his general idea and proposed a similar construction, using again Peano's idea.

It is curious that nobody noticed in this 120 years that Lebesgue in [11] (quoted in [21] for instance) refers to [10]. Also the little measure-theoretic question, which motivated it, seems to have been forgotten.

3. Homogeneity. Sierpiński and Knopp criticized the fact that in Osgood's construction some arcs have area zero and they proposed two similar constructions which eliminate this drawback, constructing two different examples of area-filling curves $\gamma$ which have only arcs of strictly positive area. We call curves with this property positive curves. Another positive curve has been constructed by Gelbaum and Olmsted (their first example).

Stromberg and Tseng [21] presented a construction of a positive area-filling curve which has an additional property.

They wrote: 'there is a 'homogeneity' feature which is desirable but not guaranteed by Knopp's and Siepiński's construction: given $\beta \in] 0,1[$, one may ask that for every subset $\mathrm{E}$ of $[0,1]$, the image $\gamma(E)$ has area equal to $\beta$ times the 'length' of $E$.

Here it is important to distinguish between curves as functions and curves as equivalence classes as we will see.

But first we want to show that an appropriate variant of the Knopp construction, which seems simpler to us, provides also this homogeneity feature.

Theorem 3.1. For every $\beta \in] 0,1[$, there exists a homogeneous construction of a Knopp curve with $\lambda_{2}([0,1])=\beta$. 

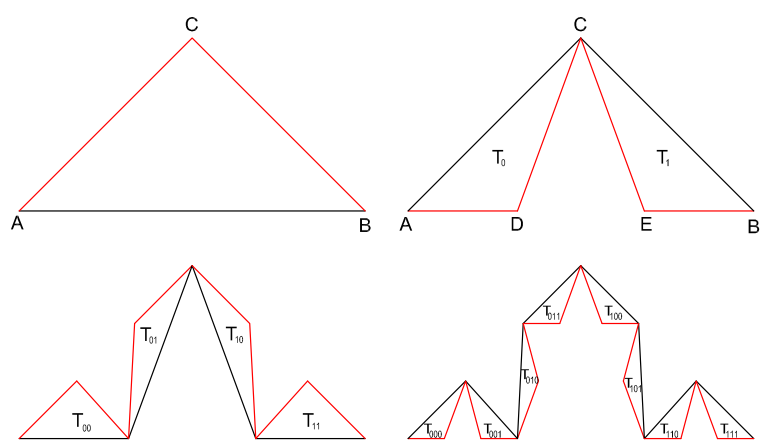

FiguRE 1. First four steps of the generation of a Knopp homogeneous area-filling curve

Proof. Take a triangle $\triangle A B C$ having area 1. At the first step subtract from it a triangle with vertex in $C$ and base $D E$ on the segment $A B$ to obtain two closed triangles $T_{0}=\triangle A D C$ and $T_{1}=\triangle E B C$ having equal area (details will be given later). See Fig. 1.

Repeat now this construction subtracting from $T_{0}$ a triangle with vertex in $D$ and base a segment on $A C$, and from $T_{1}$ a triangle with vertex in $E$ and base a segment on $B C$. This can be done obtaining a chain of four triangles (so that they have equal area) $T_{00}, T_{01}, T_{10}$, and $T_{11}$, which connects $A$ to $B$.

Iterating this procedure, we get nested chains $V_{n}$ of triangles having the same area connecting $A$ to $B$. The indices of $T_{\varepsilon_{1} \varepsilon_{2} \ldots \varepsilon_{n}}$, with $\varepsilon_{k} \in\{0,1\}$, are chosen so to establish an ordered correspondence between the triangles and the binary $n$-digit fractions $0 . \varepsilon_{1} \varepsilon_{2} \ldots \varepsilon_{n}$.

$V=\bigcap_{n=1}^{\infty} V_{n}$ is a compact connected set and for any $\left.\beta \in\right] 0,1[$, it is possible to chose (in many ways) the areas of the subtracted triangles so that $\lambda_{2}(V)=$ $\beta$.

The crucial step in Knopp's construction is to prove that the diameters of the triangles $T_{\varepsilon_{1} \varepsilon_{2} \ldots \varepsilon_{n}}$ tend to zero. This technical step proves that there exists a continuous and injective mapping $\gamma$ from $[0,1]$ onto $V$ which assigns to every real number written in the binary form $0 . \varepsilon_{1} \varepsilon_{2} \ldots \varepsilon_{n} \ldots$ the uniquely determined point $v \in V$ which belongs to the intersection $\bigcap_{n=1}^{\infty} T_{\varepsilon_{1} \varepsilon_{2} \ldots \varepsilon_{n}}$.

Our choice of the triangles is different, so we have to prove that our construction produces triangles whose diameters tend to zero, so we can use the last part of Knopp's proof.

Let $\beta \in] 0,1\left[\right.$ and take a sequence of real numbers $\left\{r_{k}\right\}$ such that $\left.r_{k} \in\right] 0,1[$ and $\prod_{k=1}^{\infty}\left(1-r_{k}\right)=\beta$.

At the first step let $T_{0}$ and $T_{1}$ have area $\frac{1-r_{1}}{2}$.

At the next step let $T_{00}, T_{01}, T_{10}$, and $T_{11}$ have area $\frac{\left(1-r_{1}\right)\left(1-r_{2}\right)}{4}$.

In general, let the $2^{n}$ triangles of $V_{n}$ have area $\frac{1}{2^{n}} \prod_{k=1}^{n}\left(1-r_{k}\right)$.

Since the sequence $\left\{r_{k}\right\}$ tends to zero, there exists $n_{0}$ such that $r_{n}<\frac{1}{8}$ for $n \geq n_{0}$. Take an $n \geq n_{0}$ and consider a triangle in the chain $V_{n}$ with vertices $N, P$, and $O$. 


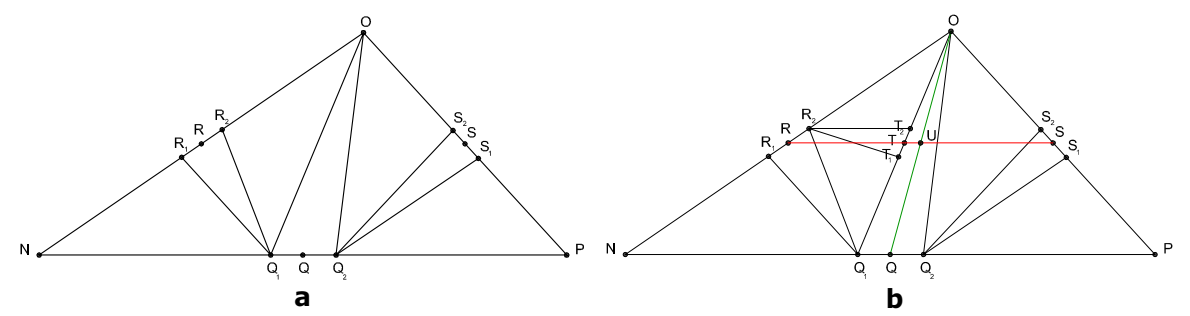

Figure 2. Triangle of $V_{n}$ and construction at the $(n+2)$ th step (left) and the $(n+3)$ th step (right)

We are going to show that in three steps the diameters of the triangles are reduced by a factor of at most $\frac{3}{4}$.

Denote by $Q$ and $R$ the midpoints of the segments $N P$ and $N O$ respectively. The triangle $\triangle N Q R$ (see Fig. 2 a)) is similar to $\triangle N P O$ with similarity ratio $\frac{1}{2}$. Since $\triangle N Q_{1} R_{1} \subset \triangle N Q R$, this triangle and all the triangles of the successive chains have diameter less than $\frac{1}{2}$ times the diameter of $\triangle N P O$. The same argument applies to $\triangle Q_{2} P S_{1}$.

Let us now turn to $\triangle R_{2} Q_{1} O$ and the operations of the next step (see Fig. 2b). $\triangle R_{2} Q_{1} O$ generates $\triangle R_{2} T_{2} O$ and $\triangle R_{2} T_{1} Q_{1}$. The first one is contained in $\triangle O R S$ and its diameter is smaller than one half of the diameter of $\triangle N P O . \triangle R_{2} Q_{1} T_{1}$ offers greater resistance. But it is easy to see that it is contained in the image of $\triangle N P O$ under a homothety which shrinks it by a factor $\frac{3}{4}$ and keeps $N$ fixed. This is due to the fact that $r_{n}<\frac{1}{8}$.

Now Knopp's argument applies and it follows that $V$ is the image of a continuous injective mapping.

Let us prove now the homogeneity property of $\gamma$. Let $T=T_{\varepsilon_{1} \ldots \varepsilon_{n}}$ be a triangle of the chain $V_{n}$ and denote by $X$ and $Y$ its vertices which connect it to the remaining part of the chain. Possibly $X=A$ or $Y=B$. The points $A$, $X, Y$, and $B$ are in that order on $V$. We have that $\{X\}=\bigcap_{m=0}^{\infty} T_{\varepsilon_{1} \ldots \varepsilon_{n} 0 \ldots 0}$, where $\varepsilon_{n}$ is followed by $m 0$ 's. Therefore $X=\gamma\left(0 . \varepsilon_{1} \ldots \varepsilon_{n}\right)$.

On the other hand $\{Y\}=\bigcap_{m=0}^{\infty} T_{\varepsilon_{1} \ldots \varepsilon_{n} 1 \ldots 1}$, where $\varepsilon_{n}$ is followed by $m$ 1's. Therefore $Y=\gamma\left(0 . \varepsilon_{1} \ldots \varepsilon_{n} \overline{1}\right)$.

It follows that the interval $I \subset[0,1]$, mapped by $\gamma$ onto the $\operatorname{arc} \widehat{X Y}$, has length $\frac{1}{2^{n}}$.

On the other hand, $T$ has area

$$
\prod_{i=1}^{n} \frac{\left(1-r_{i}\right)}{2}=\frac{1}{2^{n}} \prod_{i=1}^{n}\left(1-r_{i}\right)
$$

The $2^{m}$ triangles belonging to $V_{n+m}$ and contained in $T$ have total area

$$
2^{m} \frac{1}{2^{n+m}} \prod_{i=1}^{n+m}\left(1-r_{i}\right)=\frac{1}{2^{n}} \prod_{i=1}^{n+m}\left(1-r_{i}\right)
$$


Taking the limit for $m \rightarrow \infty$, it follows that

$$
\lambda_{2}(\widehat{X Y})=\frac{1}{2^{n}} \prod_{i=1}^{\infty}\left(1-r_{i}\right)=\beta \frac{1}{2^{n}}=\beta \lambda_{1}(I) .
$$

The equation $\lambda_{2}(\gamma(E))=\beta \lambda_{1}(E)$, for every measurable set $E$ contained in $[0,1]$, follows now from the density of binary intervals and their finite unions among measurable sets.

We may speak about a homogeneous parametrization of a curve. But curves alone cannot be considered homogeneous and we show that any positive curve can be reparametrized so to get a homogeneous parametrization.

Theorem 3.2. Let $\gamma$ be a positive area-filling curve parametrized on $[0,1]$ (with $\left.\lambda_{2}(\gamma([0,1]))=\beta\right)$. Then there exists an increasing homeomorphism $h$ from $[0,1]$ to $[0,1]$ such that $\gamma_{1}=\gamma \circ h$ is homogeneously parametrized.

Proof. The function $h^{-1}(t)=\beta^{-1} \lambda_{2}(\gamma([0, t]))$ is an increasing homeomorphism from $[0,1]$ to $[0,1]$. Let us denote its inverse with $h$.

Since $h^{-1}(h(s))=s=\beta^{-1} \lambda_{2}(\gamma([0, h(s)])$, we have

$$
\lambda_{2}\left(\gamma_{1}(s)\right)=\lambda_{2}(\gamma([0, h(s)])=\beta s
$$

as required.

On the other hand, if we take the homogeneous representation of the Knopp curve just constructed and consider the increasing homeomorphism $s(\cdot):[0,1] \rightarrow[0,1], s(t)=t^{2}$, we notice that $\gamma_{1}(t)=\gamma\left(t^{2}\right)$ is such that $\lambda_{2}\left(\gamma_{1}\left(\left[0, \frac{1}{2}\right]\right)\right)=\frac{1}{4} \beta$ and $\lambda_{2}\left(\gamma_{1}\left(\left[\frac{1}{2}, 1\right]\right)\right)=\frac{3}{4} \beta$, so $\gamma_{1}$ is not homogeneously parametrized.

4. Invasive curves. The natural question whether there exists an injective continuous curve $\gamma$ with image in $[0,1]^{2}$ such that $\lambda_{2}(\gamma([0,1]))=1$ has an immediate negative answer: $\gamma([0,1])$ is compact and the only compact subset of $[0,1]^{2}$ with measure 1 is the square itself. But this is excluded by Netto's result.

We mention here two results related to this question which can be deduced or found in [21] and [8], respectively.

Sadahiro Saeki contributed to [21] with a theorem which is not constructive since it uses the deep result due to von Neumann, Oxtoby, and Ulam, the homeomorphic measures theorem (HMT) (see [14] and [5]). He proved just the existence of non-intersecting area-filling curves. Looking at his proof, however, it is easy to draw the following (non-constructive) conclusion.

Theorem 4.1. There exist sequences $\left\{\gamma_{n}\right\}$ of non-intersecting injective curves defined on $[0,1]$ such that $\sum_{n=1}^{\infty} \lambda_{2}\left(\gamma_{n}([0,1])\right)=1$.

A result which gets closer to an answer to the question posed at the beginning of the section is contained in [8]. The authors do not state it explicitly, but it can be found in the discursive part of their article (p. 176, lines -6, -5):

Theorem 4.2. There exists a continuous and injective mapping $\gamma$ from $[0,1[$ to $[0,1]^{2}$ such that $\lambda_{2}(\gamma([0,1[))=1$.

It is an easy consequence of their nice main result, the thread theorem. 
5. Lance-Thomas curves. In the introduction, we mentioned non-constructive existence proofs. One is contained in [1]. It is not explicitly stated, but is described in the text of the proof of their Theorem 2.2. It uses the DenjoyRiesz theorem, which asserts that

Theorem 5.1. Any bounded totally disconnected set in the plane can be covered by a Jordan arc.

The existence of an area-filling curve follows easily from the previous result: fix $\beta>0$, take a planar totally disconnected set of measure $\beta$ contained in $[0,1]^{2}$, and cover it with a Jordan arc. So one obtains an area-filling curve of measure at least $\beta$ (since the Jordan arc may contain other area-filling arcs). The easiest way of producing a totally disconnected compact set in the plane of positive measure is to take a Cantor set $P \subset[0,1]$ of positive measure and to then consider $P \times P$. Having the Denjoy-Riesz theorem in mind, the proofs of Lebesgue, Osgood, and the second construction by Gelbaum and Olmsted can be immediately related to this scene, but there is no doubt that the direct inspiration came from Peano's and Hilbert's set.

It should be noted however that the first of the two constructions from [21] can also be related to this trace: fixed a $\beta>0$, they let $\alpha=\sqrt{\beta}$ and constructed a symmetric Cantor set $P \subset[0,1]$ having measure $\alpha$. Then they explicitly exhibited segments which connect points of $P \times P$ and form a Jordan arc covering $P \times P$. Its measure is $\beta$ since the segments have measure zero. The construction is explicit, but very complicated (it takes pages 34-43) and it does not present any evident symmetry. They do not provide any picture of their set and also our attempts to obtain a suggestive figure failed.

It should be noted that [1] has been published five years later, so there is no direct connection between the two papers, and we just imagine how the authors have argued.

We now make a change to the construction of the Lance-Thomas curve to fit into this line of thought. Let $\beta \in] 0,1\left[\right.$ and consider a sequence $\left\{a_{n}\right\}$ of real numbers, called the reduction coefficients, such that $0<a_{n}<1$ and $\lim _{n \rightarrow \infty}\left(a_{1} \cdot a_{2} \cdots a_{n}\right)^{2}=\beta$.

Our construction of the Lance-Thomas curve differs from the previous ones ([9], [18], and [17]) by the parametrization. Note that Sagan parametrized it on the ternary Cantor set and did not follow [9] who simply divided $[0,1]$ into seven equal parts.

To construct the first approximation, consider (following the original idea) four disjoint squares, having side length $\frac{a_{1}}{2}$, placed at the corners of $[0,1]^{2}$ and joined by three segments (joints) as in Fig. 3. The curve $\gamma_{1}$ is obtained adding to the three joints the diagonals of the squares to get a polygonal line which connects the left lower corner with the right upper corner of the square. Denote by $A_{1}$ the union of those four closed squares.

We choose to subdivide $[0,1]$ into seven subintervals so that the first, the third, the fifth, and the seventh have length $\left(\frac{a_{1}}{2}\right)^{2}$. Denote by $C_{2}$ the union of these four intervals. The second and sixth intervals have length $\frac{a_{1}}{2}\left(1-a_{1}\right)$, while the central has length $\left(1-a_{1}\right)$. The function $\gamma_{1}$ maps linearly the even 
intervals (second, fourth, sixth) in the segments that connect the squares of $A_{1}$ and the remaining subintervals in the natural order into the diagonals of the squares as shown in Fig. 3.

There is no mystery in the choice of the length of the intervals: they are equal to the areas of the seven rectangles crossed by the seven parametrized segments.

The curve $\gamma_{2}$ is constructed analogously, operating now in the four squares of the first generation using the reduction coefficient $a_{2}$. Denote by $A_{2}$ the set made up of sixteen squares obtained putting into each of the four squares of $A_{1}$ four smaller squares, placed at the vertices, of side length $\frac{a_{1}}{2} \frac{a_{2}}{2}$. Trace their junctions as in Fig. 3.

The four intervals of $C_{2}$ are subdivided as in the first step using the reduction coefficient $a_{2}$. Each interval of $C_{2}$ is subdivided into seven parts: the first, third, fifth, and seventh have length $\left(\frac{a_{1}}{2} \frac{a_{2}}{2}\right)^{2}$.

Let us denote by $C_{4}$ the union of these four disjoint closed subintervals. The second and sixth have length $\left(\frac{a_{2}}{2}\right)^{2} \cdot \frac{a_{2}}{2}\left(1-a_{2}\right)$ and the fourth has measure $\left(\frac{a_{2}}{2}\right)^{2} \cdot\left(1-a_{2}\right)$.

The function $\gamma_{2}$ maps linearly the intervals of $C_{4}$ into the diagonals of the $A_{2}$ squares and the remaining subintervals are mapped in the junction segments, as in Fig. 3, forming so a connected polygonal.

We iterate the construction, putting into each of the squares of $A_{n-1}$ four squares and three junctions as in step one and two, using at each step the reduction factor $a_{n}$. Iterating, we have $\lambda_{2}\left(A_{n}\right)=\left(a_{1} \cdot a_{2} \cdots a_{n}\right)^{2}$ and the set $C_{2 n}$ (which is the $2 n^{\text {th }}$ step of the construction of a symmetric Cantor set $C$ (see $[2,22]))$ consists of $4^{n}$ disjoint closed intervals having one-dimensional measure equal to the two-dimensional measure of the rectangle having as diagonal the segment in which the interval is mapped, so $\lambda_{1}\left(C_{2 n}\right)=\left(a_{1} \cdot a_{2} \cdots a_{n}\right)^{2}$.
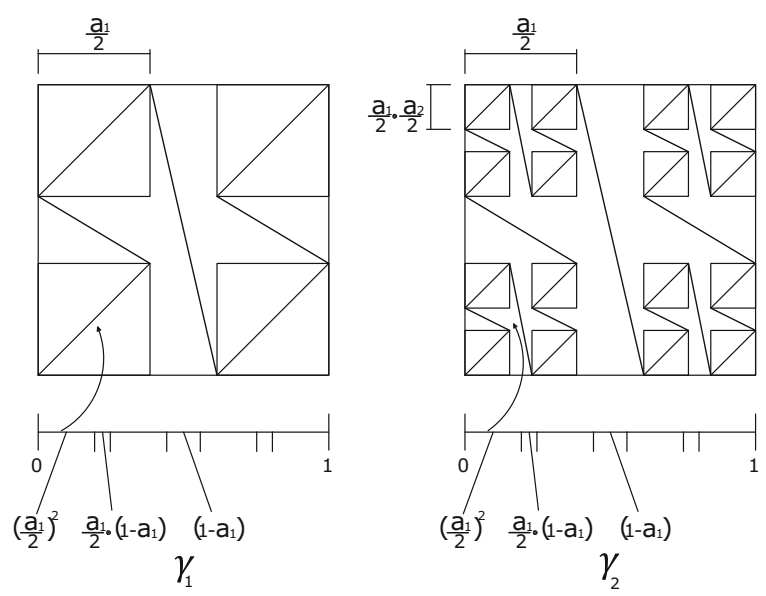

Figure 3. Construction of $\gamma_{1}$ and $\gamma_{2}$ 
Note that $\gamma_{n+1}=\gamma_{n}$ on the complement of $C_{2 n}$. On the other hand, for every $k$ and each $x \in C_{2 n},\left\|\gamma_{n+k}(x)-\gamma_{n}(x)\right\| \leq\left(\frac{a_{1}}{2} \cdot \frac{a_{2}}{2} \cdots \cdots \frac{a_{n}}{2}\right) \cdot \sqrt{2}$, which tends to 0 . Thus the sequence $\left\{\gamma_{n}\right\}$ converges uniformly to a continuous curve $\gamma$ such that

$$
\lambda_{2}(\gamma([0,1]))=\lambda_{2}\left(\bigcap_{n} A_{n}\right)=\lim _{n \rightarrow \infty}\left(a_{1} \cdot a_{2} \cdots a_{n}\right)^{2}=\beta .
$$

Remark 5.2. If we denote by $B_{n}$ the set which consists of the union of squares of $A_{n}$ for each $n$, the restriction of the limiting curve $\gamma$ to $C$ is a homeomorphism between $C$ and $B=\bigcap_{n} B_{n}$ that preserves the measure. We call the compact set $B$ the essential image of $[0,1]$ under $\gamma$.

It is easy to see that for any Borel set $E \subset[0,1], \lambda_{2}(\gamma(E \cap C))=\lambda_{1}(E \cap C)$, so our parametrization is sort of a "homogeneous" one.

Note also that $B$ is a Cartesian product of two symmetric Cantor sets contained in $[0,1]$ and that the curve $\gamma$ is a Jordan arc containing $B$. So this construction is an illustration of the Riesz-Denjoy theorem.

Open Access. This article is licensed under a Creative Commons Attribution 4.0 International License, which permits use, sharing, adaptation, distribution and reproduction in any medium or format, as long as you give appropriate credit to the original author(s) and the source, provide a link to the Creative Commons licence, and indicate if changes were made. The images or other third party material in this article are included in the article's Creative Commons licence, unless indicated otherwise in a credit line to the material. If material is not included in the article's Creative Commons licence and your intended use is not permitted by statutory regulation or exceeds the permitted use, you will need to obtain permission directly from the copyright holder. To view a copy of this licence, visit http://creativecommons.org/ licenses/by/4.0/.

Publisher's Note Springer Nature remains neutral with regard to jurisdictional claims in published maps and institutional affiliations.

\section{References}

[1] Balcerzak, M., Kharazishvili, A.: On uncountable unions and intersections of measurable sets. Georgian Math. J. 6(3), 201-212 (1999)

[2] Falconer, K.J.: The Geometry of Fractal Sets. Cambridge University Press, Cambridge (1985)

[3] Fréchet, M.: Sur quelques points du calcul fonctionnel. Rend. Palermo 27, 1-74 (1906)

[4] Gelbaum, B.R., Olmsted, J.M.H.: Counterexamples in Analysis. The Mathesis Series Holden-Day, Inc., San Francisco, Calif.-London-Amsterdam (1964)

[5] Goffman, C., Pedrick, G.: A proof of the homeomorphism of Lebesgue-Stieltjes measure with Lebesgue measure. Proc. Amer. Math. Soc. 52, 196-198 (1975)

[6] Hilbert, D.: Über die stetige Abbildung einer Linie auf ein Flächenstück. Math. Ann. 38(3), 459-460 (1891) 
[7] Knopp, K.: Einheitliche Erzeugung und Darstellung der Kurven von Peano, Osgood und von Koch. Arch. Math. Phys. 26, 103-115 (1917)

[8] Hajłasz, P., Strzelecki, P.: How to measure volume with a thread. Amer. Math. Monthly 112, 176-179 (2005)

[9] Lance, T., Thomas, E.: Arcs with positive measure and a space-filling curve. Amer. Math. Monthly 98(2), 124-127 (1991)

[10] Lebesgue, H. Intégrale, longueur, aire. Thesis, Faculté des Sciences de Paris (1902)

[11] Lebesgue, H.: Sur le problème des aires. Bull. Soc. Math. France 31, 197-203 (1903)

[12] Netto, E.: Beitrag zur Mannigfaltigkeitslehre. J. Reine Angew. Math. 86, 263$268(1879)$

[13] Osgood, W.F.: A Jordan curve of positive area. Trans. Amer. Math. Soc. 4(1), 107-112 (1903)

[14] Oxtoby, J.C., Ulam, S.M.: Measure-preserving homeomorphisms and metrical transitivity. Ann. Math. (2) 42, 874-920 (1941)

[15] Peano, G.: Sur une courbe, qui remplit toute une aire plane. Math. Ann. 36(1), 157-160 (1890)

[16] Ravishankar, K., Salas, H.: On the existence of locally heavy arcs. Rev. Un. Mat. Argentina 36(1990), 101-110 (1992)

[17] Sagan, H.: A geometrization of Lebesgue's space-filling curve. Math. Intelligencer 15(4), 37-43 (1993)

[18] Sagan, H.: Space-Filling Curves. Universitext, New York (1994)

[19] Sergeyev, Y.D., Strongin, R.G., Lera, D.: Introduction to Global Optimization Exploiting Space-Filling Curves. Springer, Berlin (2013)

[20] Sierpiński, W.: Sur une courbe non quarrable. Bull. Acad. Sci. de Cracovie Sci. math. et nat., Série A, 254-263 (1913)

[21] Stromberg, K., Tseng, S.: Simple plane arcs of positive area. Expo. Math. 12, 31-52 (1994)

[22] Vallin, R.W.: The Elements of Cantor Sets with Applications. Wiley, New York (2013)

\section{Maria Chiara Nasso}

Dipartimento di Ingegneria Informatica, Modellistica, Elettronica e Sistemistica

Università della Calabria

Rende CS

Italy

e-mail: mc.nasso@dimes.unical.it 


\author{
AlJoša VOLČIČ \\ Dipartimento di Matematica e Informatica \\ Università della Calabria \\ Rende CS \\ Italy \\ e-mail: aljosa.volcic@unical.it
}

Received: 13 October 2021

Revised: 9 December 2021

Accepted: 6 January 2022. 